\title{
THE INDUCTION BY ARSENIOUS ACID OF THE REACTION BETWEEN CHROMIC AND HYDRIODIC ACIDS
}

\section{BY RALPH LE. DE LURY}

It has long been known that the process of oxidation in solution can often be hastened by the presence of a small quantity of some easily oxidizable substance. This phenomenon is called induced oxidation: the name acceptor is given to the substance whose oxidation is accelerated and the reagent causing the acceleration is called the inductor. Ferrous salts, arsenious acid and nitric oxide act as inductors and iodide as acceptor for the oxygen of chromic acid.

About the niliddle of the last century many cases of induced oxidation, occurring chiefly in the development of methods of analysis, were noted and classified by Schönbein. Kessler and others. By selecting various combinations of reducing and oxidizing agents the number of similar cases might be multiplied indefinitely. Many of these cases have been studied qualitatively and to a certain degree quantitatively, and the attempts to explain the results of these investigations have led to the development of the peroxide. theory so ably championed by Marichot. ${ }^{1}$

The peroxide theory is based on a considerable amount of qualitative and some quantitative data, and on the fact that some peroxides are known to be formed in the process of oxidation, such as hydrogen peroxide and benzoyl hydroperoxide: these peroxides may be regarded as the primary products of the oxidation of hydrogen and benzaldehyde respectively.

However, if a non-isolable peroxide, regarded as the cause of induced oxidation, be formed, its formation can be ascertained only by a careful study of the rates of all the reactions involved, since it is as an explanation of these rates - ${ }^{1}$ Lieb. Ann., 325, 95 (1902). 
that the peroxide theory is advanced. This obvious method of attacking the problem has been neglected, no doubt on account of the difficulties of analysis which such a method in general presents; hence the quantitative investigation of such cases has been chiefly confined to the measurement of the ratio of the amounts of inductor and acceptor oxidized when the reaction is complete. ${ }^{1}$ Such a method will often give the oxidizing value of the intermediate product but the results are not reliable or sufficient.

The only case of which I am aware to which the method of studying all the rates involved has been applied as yet, is the reactions in solutions containing chromic acid, iodide and ferrous salt ${ }^{2}$ investigated by Miss C. C. Benson, and curiously enough the results are not in accord with the peroxide theory.

In the present paper I communicate the results of measurements of the rates of the reactions in solutions containing chromic acid, iodide and arsenious acid and am able to show that the assumption of the formation of a peroxide explains the induction in this case. The rates of oxidation of iodide ${ }^{3}$ and of arsenious acid ${ }^{4}$ by chromic acid have already been studied by myself.

\section{Plan of the Investigation}

In solutions containing potassium iodide, arsenious acid, potassium bichromate and sulphuric acid the following reactions may occur:

$A$. Oxidation of the iodide by chromic acid,

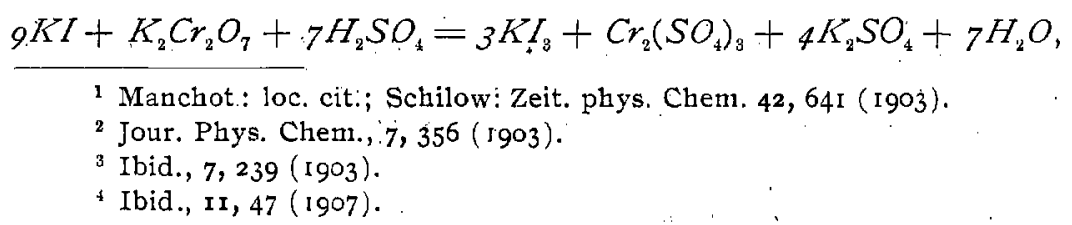


whose rate at $0^{\circ} C$, may be represented ${ }^{1}$ approximately by the kinetic equation: ${ }^{2}$

$$
\frac{d I_{3}}{d t}(c)=R i c=I \times I O^{-12} \cdot C r \cdot(A c)^{2} \cdot\left[K I+0.002(K I)^{2}\right]
$$

$B$. Oxidation of arsenious acid by chromic acid, $3 \mathrm{As}_{2} \mathrm{O}_{3}+2 \mathrm{~K}_{2} \mathrm{Cr}_{2} \mathrm{O}_{7}+8 \mathrm{H}_{2} \mathrm{SO}_{4}=3 \mathrm{As}_{2} \mathrm{O}_{5}+2 \mathrm{Cr}_{2}\left(\mathrm{SO}_{4}\right)_{3}+2 \mathrm{~K}_{2} \mathrm{SO}_{4}+8 \mathrm{H}_{2} \mathrm{O}$. In the absence of potassium iodide the rate of this reaction at $0^{\circ} \mathrm{C}$, may be represented by the equation: ${ }^{3}$

$$
\frac{d A s}{d t}(c)=R a c=83 \times I 0^{-8} \cdot A s \cdot(C r)^{0.9} \cdot(A c)^{\top \cdot 4}
$$

C. Oxidation of the iodide by arsenic acid formed in the reaction,

$$
6 \mathrm{KI}+\mathrm{As}_{2} \mathrm{O}_{5}+2 \mathrm{H}_{2} \mathrm{SO}_{4}=2 \mathrm{KI}_{3}+\mathrm{As}_{2} \mathrm{O}_{3}+2 \mathrm{~K}_{2} \mathrm{SO}_{4}+2 \mathrm{H}_{2} \mathrm{O},
$$

and the rate of this reaction at $0^{\circ} C$, may be represented ${ }^{4}$ thus:

$$
\frac{d I_{3}}{d t}(a)=R i a=4 \times I O^{-12} \cdot A s \cdot K I . A c
$$

$1 K I, C r$ and $A C$ denote the amounts of potassium iodide, potassium bichromate and sulphuric acid respectively, expressed in 10-5 gram-formulaweights (i. e. in $c c$. of o.or $F$ solution): the same unit as employed in the measurenents on the rate of oxidation of iodide by chromic acid (loc. cit.). As and As denote respectively the amounts of arsenious acid and arsenic acid expressed in $0.25 \times$ 10-5 gram-formula-weights (i. e. in $c c$. of 0.0 r $N$ solution); this unit is selected in order to facilitate comparison between the iodine and arsenious acid readings in the Tables which follow. $I_{3}$ denotes the "free iodine" in $0.5 \times$ 1o-5 gram-formula-weights (i. e. in $c c$. of $0.01 N$ solution). The letter $R$ denotes rate; $R i c$ denotes the rate of oxidation of iodide by chromic acid; Rac denotes the rate of oxidation of arsenious acid by chromic acid, etc. In the subscripts $i c, a c$, etc. the letters $i, a, c$, suggest iodide (or triiodide), arsenious acid (or arsenic acid), and chromic acid respectively: the letter which goes first denoting the substance oxidized and the second letter the substance oxidizing.

The constants are calculated for a volume of $300 \mathrm{cc}$.

${ }^{2}$ De Lury: loc. cit.

3 The paper just preceding the present paper.

4 Roebuck: Jour. Phys. Chem., 6, 365 (1902). In concentrated solutions the indices of the power of $K I$ and $A C$ are greater than $\mathrm{I}$. 
$D$. Oxidation of the arsenious acid by the triiodide formed in the reaction,

$$
\mathrm{As}_{2} \mathrm{O}_{3}+2 \mathrm{KI}_{3}+2 \mathrm{~K}_{2} \mathrm{SO}_{4}+2 \mathrm{H}_{2} \mathrm{O}=\mathrm{As}_{2} \mathrm{O}_{5}+6 \mathrm{KI}+{ }_{2} \mathrm{H}_{2} \mathrm{SO}_{4}
$$

whose rate at $0^{\circ} C$, is expressed by : $:^{1}$

$$
\frac{d A^{v}}{d t}(i)=-\frac{d I_{3}}{d t}(a)=R a i=I \times I O^{-6} \cdot I_{3} \cdot A s \cdot(K I)^{-2} \cdot(A c)^{-1} \text { (4) }
$$

$E$. Oxidation of the iodide due to the influence of the arsenious acid; the symbol Rip denotes the rate of this reaction. $^{2}$

In order to simplify the study of the rate of reaction $E$ the concentrations of the reagents were selected so as to reduce the rates of reactions $A, C$ and $D$ as much as possible. Of these, reaction $C$ (Ria) gave no trouble at all since the rate constant is very small and no arsenic acid was present except that formed in reactions $B$ (Rac) and $D$ (Rai). The loss of $I_{3}$ by reaction $D$ could not be prevented by high concentrations of $K I$ and $A c$ without unduly increasing Ric, and in the end it was found necessary to remove the iodine as soon as it was liberated by the use of thiosulphate (see page 58 ).

Thus in my experiments Ria and Rai were negligibly small, and therefore,

$$
\begin{aligned}
& \frac{d A s}{d t}=R a=R a c+M^{3} \\
& \frac{d I_{3}}{d t}=R i=R i c+R i p
\end{aligned}
$$

and Ric being determined by blank experiments Rip is obtained by difference.

\section{EXPERIMENTAL PART}

It was necessary to determine the quantities of iodide and arsenious acid oxidized at any stage of the reaction. Three methods of analysis were tried.

First Method.-When a suitable interval had elapsed the

1 Roebuck: Loc. cit.

${ }^{2} p$, for peroxide in alticipation of the theory (see p. $7^{8}$ ).

${ }^{3} M$ is the modification in $R a c$ due to the iodide (see p. 73 ). 
concentration of the acid was reduced by addition of sodium bicarbonate, the iodine quickly titrated with sodium thiosulphate, excess of ammonium bicarbonate added and the residual arsenious acid titrated with iodine. This method was not successful.

Second Method.-After the reaction had proceeded for some time excess of ammonium bicarbonate was added and the arsenious acid titrated with iodine. In a duplicate experiment after the same interval, a measured quantity of $0.002 \mathrm{~N}$ thiosulphate solution, slightly in excess of the iodine present, was thrown into the reacting mixture, with violent stirring just before the ammonium bicatbonate which served as restrainer: Starch was then added and the arsenious acid and residual thiosulphate titrated with iodine, the iodine added being the sum of the arsernious acid and a function of the remaining thiosulphate. From these two measurements the arsenious acid oxidized and the iodine present could be determined if this function were known. Experiments showed that it depended on so many variables that no reliable determinations could be made, and the method was abandoned.

Third Method.-In the end a modification of Harcourt and Fisson's compensation method was found to serve, and was adopted in all the measurements of this paper. The details of the method are as follows:

\section{Method of Making the Meașurements}

Distilled water and dilute solutions of potassium bichromate, sulphuric acid, potassium iodide and arsenious acid were kept at $0^{\circ} \mathrm{C}$ in a thermostat. The bichromate, sulphuric acid and water were mixed in a beaker coated on the outside with white enamel. In a large test-tube the iodide and arsenious acid were mixed and a little starch solution added. At a suitable time the contents of the test-tube were thrown into the beaker with violent stirring, and a stopwatch set 'going' by pressure of the foot.

Over the beaker was clamped a ro $c c$ burette from which o.or $N$ sodium thiosulphate solution was added drop by drop 
as the reaction proceeded, care being taken to keep the solution in the beaker as near as possible free from both iodine and thiosulphate. A number of readings of the burette were made during each experiment, and finally after a definite interval the reaction was stopped with excess of ammonium bicarbonate, and the residual arsenious acid titrated immediately ${ }^{1}$ with iodine.

To test the method of analysis the measurements recorded in Tables I, II and III were made. In these tables, "yellow" denotes "slight excess of thiosulphate," and "green" denotes "slight excess of iodine," that is, just enough to keep the reacting mixture bluish green with the starch present; "close" means "as near as possible free from both thiosulphate and iodine."

TABLE I

TABLE II

$A s$, 10.00; $K I, 9.8 ; C r, 20 ; A c, 484 A s, 10.00 ; K I, 245 ; C r, 20 ; A c, 242$

\begin{tabular}{c|cc|l} 
Minutes & $c c$ thiosulphate & Minutes & $c c$ thiosulphate \\
\hline 5.1 & I.59 yellow & 9.5 & 2.00 yellow \\
5.1 & I.31 close & 9.5 & 2.00 close \\
5.1 & I.09 green & 9.6 & 2.00 green
\end{tabular}

TABLE III

As, $10.00 ; K I, 98 ; C r, 20 ; A c, 242$

\begin{tabular}{c|c|c|c}
\multirow{2}{*}{ cc thiosulphate } & ycllow & close & green \\
\cline { 2 - 3 } & Minutes & Minutes & Minutes \\
\hline 0.53 & 3.2 & 3.5 & 3.3 \\
1.00 & 6.0 & 6.2 & 6.2 \\
1.50 & 9.0 & 9.1 & 8.8 \\
2.00 & I 1.9 & I 2.0 & 12.0
\end{tabular}

In Table I where the concentration of the iodide was low the amount of thiosulphate needed to neutralize the iodine liberated in a fixed interval, depends on the method of working. This is due to the absorption of iodine by arsenious acid, and as the rate of this reaction ${ }^{2}$ is inversely proportional

1 To prevent oxidation of the arsenite in the alkaline solution of chromate and oxygen.

${ }^{2}$ See equation (4). 
to the square of the concentration of the iodide, the difference in the thiosulphate readings becomes less when the concentration of the iodide is increased and disappears when the iodide concentration is equal to (or greater than) ten times the con-. centration of the arsenious acid (Tables II and III). The fact that in these last two tables, the readings made when the mixture was "yellow" and "green" are the same is proof that the oxidation of thiosulphate by chromic acid ${ }^{1}$ is negligible. In those of ny measurements where the concentration of the iodide was small, I was careful to keep the reacting mixture slightly "yellow" until near the end of the interval desired; this involved an approximate knowledge of the rate at which the iodine was being liberated and in some cases necessitated the duplication of the experiments.

A number of blank experiments carried out for the purpose, showed that neither the oxygen of the air, nor the arsenic acid ${ }^{2}$ and tetrathionate ${ }^{3}$ formed in the reaction, affected the rates.

\section{Solutions Employed}

In addition to the solutions, $\mathrm{K}_{2} \mathrm{Cr}_{2} \mathrm{O}_{7}$, O.I $\mathrm{F}$ or $0.6 \mathrm{~N}$; $\mathrm{H}_{2} \mathrm{SO}_{4}$, approximately o.I $\mathrm{N} ; \mathrm{As}_{2} \mathrm{O}_{3}, 0.025 \mathrm{~F}$ or O.I $\mathrm{N}$; ammonium bicarbonate and starch, employed in the neasurements on the rate of oxidation of arsenious acid by chromic acid, a solution of potassium iodide $0.098 F$ (standardized by silver) and approximately o.0I $N$ solutions of thiosulphate and iodine (standardized by comparison with the arsenious acid), were used.

\section{Explanation of the Tables}

After $K I, C r$ and $A c$ at the head of the tables are given the amounts, expressed in $10^{-5}$ gram-formula-weights $(i . e$., in $c c$ of o.oI $F$ solution) of $\mathrm{KI}, \mathrm{K}_{2} \mathrm{Cr}_{2} \mathrm{O}_{7}$ and $\mathrm{H}_{2} \mathrm{SO}$, respectively

I See Bell: Jour. Phys. Chem., 7, 6i (rgo3).

" See equation (3) above, et seq.

3. The concentration of the tetrathionate is not large enough to cause any error due to decomposition in the alkaline solution during the short interval between adding the bicarbonate and the completion of the titration. 
(the same unit as employed in the measurements of the oxidation of iodide); after $A s$, the amount of $A s_{2} O_{3}$, the unit being $0.25 \times 1 \mathrm{IO}^{-5}$ gram-formula-weights $(i . e$. , I $c c$ o.OI $N$ solution), this unit is selected so as to facilitate comparison between the iodine and arsenious acid readings.

Under $t$ are entered the durations of the reaction in minutes. Under $h$ are given the amounts of thiosulphate ("hypo"), added from the small burette during the reaction expressed in $c c$ of $0.01 N$ solution. Under $A s-x^{\prime}$ are entered the amounts of arsenious acid remaining unoxidized after $t$ minutes, expressed in $c c$ of 0.0 I $N$ solution; $x^{\prime}$ thus represents the amount of arsenious acid oxidized: this symbol is used only when iodide is present in the reacting mixture. The symbol $x$ denotes the amount of arsenious acid oxidized in the absence of iodide.

Under $i c$ in Table XXI are given the amounts of iodine (in $c c$ of $0.01 N$ solution) liberated by the oxidation of iodide in the absence of arsenious acid. The experiments were carried out just like those in which arsenite was present (page 58 ), and the $i c$ readings were obtained from the readings of the small thiosulphate burette. The numbers under $i c$ in Tables IV-X and XII-XX were obtained from these by interpolation. In Table XI where the reaction was allowed to proceed until the arsenious acid was completely oxidized, the numbers under ic (calc) were calculated from the equation, $i c($ calc $)=0.60 \frac{t}{5} \cdot \frac{20 \times 6-0.5\left(h+x^{\prime}\right)}{20 \times 6}$, thus allowing for the decrease in the concentration of the bichromate.

In all of the experiments the initial volume was as near as possible $300-\frac{h}{2} c c$, so that the average volume during the reaction was $300 \quad c c$. The temiperature was always $0^{\circ} \mathrm{C}$ except in Table XX and in one series of Table XXI where the temperature was $10^{\circ} \mathrm{C}$.

The difference between the amourits of iodine liberated in the presence and absence of arsenious acid is entered under 
$h$-ic. The constant, $k^{\prime}$, is defined by the equation, $k^{\prime}=$ $\because \frac{I}{t} \log _{10} \frac{A s}{A s-x^{\prime}}$. When no iodide is present $k^{\prime}$ becomes $k$ of the tables on the oxidation of arsenious acid by chromic acid, and at the same time $x^{\prime}$ becomes $x$ of those tables (i. e., $k$ and $x$ are the limiting values of $k^{\prime}$ and $x^{\prime}$ when $K I=0$ ).

In Tables V-IX, $R a$ and $R$ ip (see page 56 ) represent the rates of oxidation of arsenious acid, and of iodide in the presence of arsenious acid after correcting for the direct action of chromic acid. Since the concentrations of the bichromate and sulphuric acid are so large that they may be regarded as constant, the rate, $R a$, may be calculated by the equation:

$$
R a=2.30 k^{\prime} . A s
$$

$R a c$ is the limiting value of $R a$ when $K I=0$.

A rough approximation to the rate, $R i p$, is given by the quotient, $\frac{h-i c}{t}$; in this expression, however, the decrease in the concentration of the arsenious acid during the reaction is neglected. In the experiments of Tables $V_{-}-$IX this decrease is at most 25 percent, and hence the average amount of arsenite present during the interval is closely represented by $A s-0.5 x^{\prime}$. On page $74 \mathrm{I}$ show that the rate is proportional to the concentration of the arsenious acid, and have therefore employed the following expression for calculating the values of $R i p$ :

$$
R i p=\frac{h-i c}{t} \cdot \frac{A s}{A s-0.5 x^{\prime}} .
$$

TABLE IV

As, $9.94 ; K I, n .9 .8 ; C r, 20 ; A c$, I2 I; $t, 22.5$

\begin{tabular}{r|c|c|c|c|c|c|c}
\hline$n$ & $h$ & $A s-x^{\prime}$ & $x^{\prime}$ & $i c$ & $h-i c$ & $r=\frac{h-i c}{x^{\prime}}$ & $k^{\prime}$ \\
\hline 30 & 1.60 & 9.25 & 0.69 & 0.18 & 1.42 & 2.06 & 0.0014 \\
40 & 1.75 & 9.30 & $0.64 \cdot$ & $0.3 \mathrm{I}$ & $\mathrm{I} .44$ & 2.25 & 0.0013 \\
$5 \mathrm{O}$ & 1.93 & 9.26 & 0.68 & 0.52 & $\mathrm{I} .4 \mathrm{I}$ & 2.07 & 0.0013 \\
$6 \mathrm{O}$ & 2.03 & 9.33 & $0.6 \mathrm{I}$ & 0.70 & $\mathrm{I} .33$ & 2.18 & 0.0012 \\
70 & 2.25 & 9.28 & 0.66 & 0.90 & 1.35 & 2.04 & 0.0013 \\
80 & 2.40 & 9.27 & 0.67 & $\mathrm{I} .10$ & 1.30 & 1.94 & 0.0013 \\
\hline
\end{tabular}


The Induction by Arsenious Acid, Etc.

\section{TABLE V}

$A s, 9.94 ; K I, n .9 .8 ; C r, 20 ; A c, 242 ; t$, го

\begin{tabular}{|c|c|c|c|c|c|c|c|c|}
\hline$n$ ! & $h$ & $A s-x^{\prime}$ & $i c$ & $r=\frac{h-i c}{x^{\prime}}$ & $k^{\prime}$ & $R i p$ & $R a$ & $R i p+R a$ \\
\hline 0 & - & - & - & - & 一 & 0.000 & 0.263 & 0.263 \\
\hline I & 0.43 & 7.97 & O.OI & $0.2 \mathrm{I}$ & 0.0096 & 0.046 & $0.22 \mathrm{I}$ & 0.267 \\
\hline 2 & 0.70 & 8.32 & 0.02 & 0.42 & 0.0077 & 0.073 & 0.178 & $0.25^{\mathrm{I}}$ \\
\hline 4 & I. 33 & 8.79 & 0.04 & I. 12 & 0.0053 & 0.137 & 0.122 & 0.259 \\
\hline$\frac{7}{6}$ & 1.53 & $8.8 \mathrm{I}$ & 0.06 & 1.30 & 0.0052 & o. I 56 & 0.120 & 0.276 \\
\hline 8 & 1.60 & 8.86 & 0.08 & $1.4 \mathrm{I}$ & 0.0050 & 0.160 & O. I 16 & 0.276 \\
\hline Io & I. 7.4 & .9 .00 & 0.10 & ง.75 & 0.0043 & 0.172 & 0.099 & $0.27 \mathrm{I}$ \\
\hline rol. & I.98 & 9.16 & 0.10 & $(2.4 \mathrm{I})$ & 0.0036 & 0.195 & 0.083 & 0.278 \\
\hline I 2 & 1.80 & 9.07 & 0.14 & 1.91 & 0.0040 & 0.1 .73 & 0.092 & 0.265 \\
\hline 15 & 1.83 & $9 \cdot 12$ & 0.19 & 2,00 & 0.0037 & 0.170 & 0.085 & 0.255 \\
\hline 20 & I. 88 & 9.00 & 0.26 & $(\mathrm{i} .7 \mathrm{I})$ & 0.0043 & o. 169 & 0.099 & 0.268 \\
\hline 30 & 2.00 & 9. 18 & 0.43 & 2.07 & 0.0035 & 0.163 & $0.08 \mathrm{I}$ & 0.244 \\
\hline 30 & 2. 17 & 9.12 & 0.43 & 2.12 & 0.0037 & O. I 82 & 0.085 & 0.267 \\
\hline 40 & 2.24 & 9.25 & 0.63 & 2.3 .3 & $0.003 \mathrm{I}$ & o. 167 & $0.07 \mathrm{I}$ & $0.23^{8}$ \\
\hline 50 & $2.5^{8}$ & 9.29 & 0.95 & $(2.5 I)$ & 0.0029 & 0.168 & 0.067 & 0.235 \\
\hline 60 & 2.70 & 9.18 & I. I 5 & $2: 04$ & 0.0035 & 0.160 & $0.08 \mathrm{I}$ & 0.241 \\
\hline 70 & 3.02 & 9.25 & 1.45 & 2.27 & $0.003 \mathrm{I}$ & 0.162 & $0.07 \mathrm{I}$ & 0.233 \\
\hline 801 & 3.30 & 9.26 & 1.75 & 2.26 & $0.003 \mathrm{I}$ & 0.160 & $0.07 \mathrm{I}$ & 0.231 \\
\hline
\end{tabular}

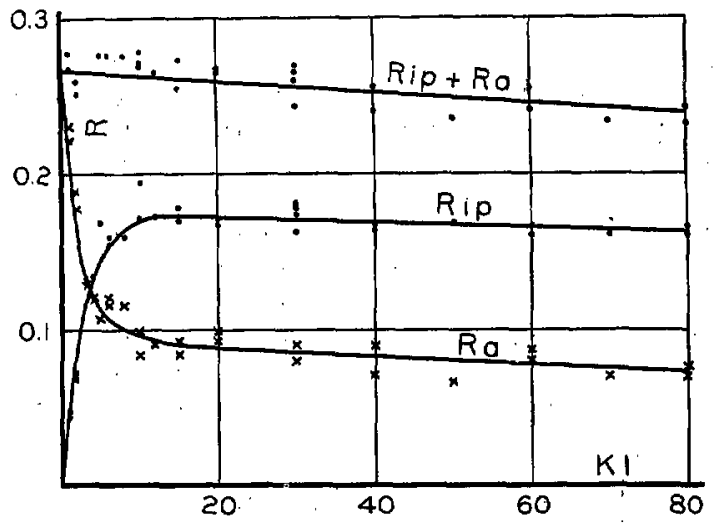

Fig. 1 (Cf. Tables V and VI)

NOTE.-In Figs. $1-4$, the unit in which $K I$ is represented is $9.8 \times 10^{-5}$ gram-formula-weights. 
TABLE VI

As, 9.94;KI, n.9.8; Cr, 20;Ac, 242; t, 15

\begin{tabular}{|c|c|c|c|c|c|c|c|c|}
\hline$n 1$ & $h$ & $A s-x^{\prime}$ & $i c$ & $r=\frac{h-i c}{x^{\prime}}$ & $k^{\prime}$ & $R i p$ & $R a$ & $R i p+R a$ \\
\hline $\mathbf{O}^{1}$ & -- & - & 一 & - & - & 0.000 & 0.263 & 0.263 \\
\hline I! & 0.63 & 7.03 & 0.01 & $0.2 \mathrm{I}$ & O.OIOO & 0.047 & 0.230 & 0.277 \\
\hline 2 & 0.95 & $7 \cdot 4^{8}$ & 0.02 & $0.3^{8}$ & 0.0082 & 0.069 & o. 189 & 0.258 \\
\hline 3 & I. 87 & 8.15 & 0.03 & 1.03 & 0.0057 & 0.134 & O.I3 I & 0.265 \\
\hline 5 & 2.40 & 8.46 & 0.05 & I.59 & 0.0047 & 0.169 & 0.108 & 0.277 \\
\hline 6 & 2.27 & 8.33 & 0.06 & (I.37) & 0.005 I & 0.160 & 0.117 & 0.277 \\
\hline Io & $2.5^{2}$ & 8.56 & O. I 5 & I.68 & 0.0043 & 0.170 & 0.099 & 0.269 \\
\hline I 5 & 2.72 & 8.6 .3 & 0.28 & I. 86 & $0.004 \mathrm{I}$ & 0.179 & 0.094 & 0.273 \\
\hline 20 & 2.78 & 8.63 & 0.39 & I. 82 & $0.004 \mathrm{I}$ & 0.171 & 0.094 & 0.265 \\
\hline 30 & 3.15 & 8.67 & 0.64 & I.98 & 0.0040 & 0.178 & 0.092 & 0.270 \\
\hline 30 & 3. II & 8.74 & 0.64 & 2.06 & 0.0037 & 0.175 & 0.085 & 9.260 \\
\hline 40 & $3 \cdot 34$ & 8.69 & 0.95 & I.9 I & 0.0039 & 0.166 & 0.090 & 0.256 \\
\hline 60 & 4.00 & 8.73 & I.66 & I.93 & 0.0038 & 0.166 & 0.088 & 0.253 \\
\hline 80 & 5.00 & 8.84 & 2.62 & 2.14 & 0.0033 & 0.166 & 0.076 & 0.242 \\
\hline
\end{tabular}

TABLE VII

$A s, 19.90 ; K I, n .9 .8 ; C r, 20 ; A C, 242 ; t$, 10

\begin{tabular}{|c|c|c|c|c|c|c|c|c|}
\hline$n$ & $h$ & $A s-x^{\prime}$ & $i c$ & $r=\frac{h-i c}{x^{\prime}}$ & $k^{\prime}$ & $R i p$ & $R a$ & $R i p+R a$ \\
\hline o & - & - & - & - & - & 0.000 & 0.536 & 0.536 \\
\hline 4 & 2.85 & 17.63 & 0.04 & I. 24 & 0.0053 & 0.296 & 0.243 & 0.539 \\
\hline 6 & 3.05 & $\mathrm{I} 7.79$ & 0.06 & I. $4 \mathrm{I}$ & 0.0049 & $0.3 I_{4}$ & 0.224 & $0.53^{8}$ \\
\hline 8 & 3.10 & I 7.77 & 0.08 & I. $4 \mathrm{I}$ & 0.0049 & 0.317 & 0.227 & 0.544 \\
\hline IO & 3.20 & 17.83 & 0.10 & I. 50 & 0.0048 & 0.325 & 0.220 & 0.545 \\
\hline I 2 & 3.30 & 18.03 & O.I4 & $1.5 \mathrm{I}$ & 0.0043 & 0.330 & 0.198 & 0.534 \\
\hline 15 & $3 \cdot 44$ & 18.07 & 0.19 & 1.77 & 0.0042 & 0.340 & 0.194 & 0.534 \\
\hline 20 & 3.57 & 18.12 & 0.26 & 1.86 & 0.0641 & $0.34^{6}$ & 0.188 & 0.534 \\
\hline 30 & 3.76 & I 8.26 & 0.43 & 2.05 & 0.0037 & 0.347 & 0.172 & 0.519 \\
\hline 50 & 4.40 & I 8.27 & 0.95 & 2. I 2 & 0.0037 & 0.359 & O. 172 & $0.53 \mathrm{I}$ \\
\hline $70^{\prime}$ & 4.80 & I $8.3 \mathrm{I}$ & I. 45 & 2. I I & 0.0036 & $0.34^{8}$ & 0.167 & 0.5 I 5 \\
\hline
\end{tabular}

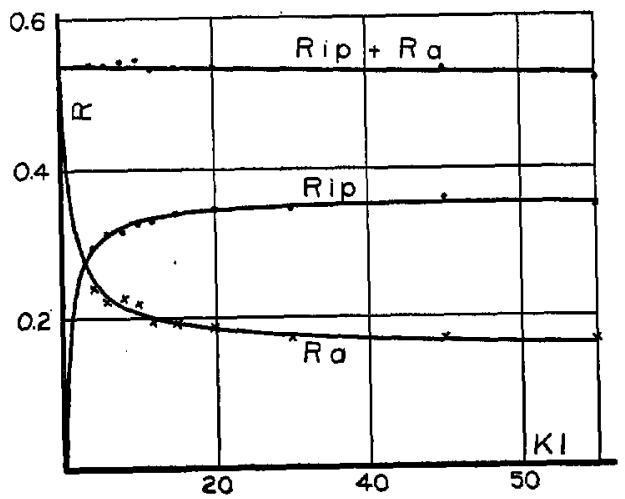

Fig. 2 (Cf. Table VII) 


\section{TABLE VIII}

$A s, 9.94 ; K I, n .9 .8 ; C r, 40 ; A c, 242 ; t$, Iо

\begin{tabular}{r|c|c|c|c|c|c|c|c|c}
\multicolumn{1}{r|}{$n$} & $h$ & $A s-x^{\prime}$ & ic & $r=\frac{h-i c}{x^{\prime}}$ & $k^{\prime}$ & $R i p$ & $R a$ & $R i p+R a$ \\
\hline 0 & - & - & - & - & & - & 0.000 & 0.500 & 0.500 \\
5 & 2.87 & 8.12 & 0.09 & 1.53 & 0.0088 & 0.303 & 0.202 & 0.505 \\
10 & 3.10 & 8.28 & 0.18 & 1.76 & 0.0079 & 0.315 & 0.183 & 0.498 \\
I.5 & 3.26 & 8.34 & 0.33 & 1.83 & 0.0076 & 0.316 & 0.176 & 0.492 \\
20 & 3.55 & 8.35 & 0.48 & 1.93 & 0.0075 & 0.331 & 0.175 & 0.506 \\
30 & 3.85 & 8.35 & 0.80 & 1.93 & 0.0076 & 0.329 & 0.175 & 0.504 \\
50 & 4.82 & 8.40 & 1.80 & 2.00 & 0.0073 & 0.326 & 0.168 & 0.494
\end{tabular}

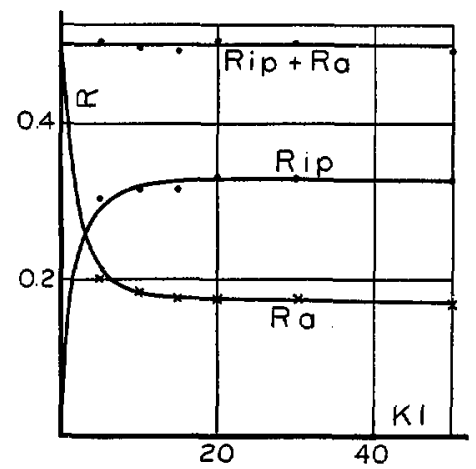

Fig. 3 (Cf, Table VIII)

TABLE IX

As, 9.94; KI, n.9.8; Cr, 20;Ac, 484; t, 10

\begin{tabular}{r|c|c|c|c|c|c|c|c}
\hline \multicolumn{1}{r|}{$n$} & $h$ & $A s-x^{\prime}$ & ic & $r=\frac{h-i c}{x^{\prime}}$ & $k^{\prime}$ & $R i p$ & $R a$ & $R i p+R a$ \\
\hline 0 & - & - & - & - & - & 0.000 & 0.765 & 0.765 \\
5 & 4.59 & 7.12 & 0.28 & 1.54 & 0.0183 & 0.487 & 0.306 & 0.793 \\
10 & 5.06 & 7.32 & 0.56 & 1.72 & 0.0133 & 0.509 & 0.306 & 0.815 \\
IO & 5.23 & 7.53 & 0.56 & 1.94 & 0.0121 & 0.525 & 0.279 & 0.804 \\
I 5 & 5.54 & 7.58 & 0.84 & 2.00 & 0.0118 & 0.525 & 0.272 & 0.797 \\
20 & 6.00 & 7.81 & 1.20 & 2.25 & 0.0105 & 0.531 & 0.242 & 0.773 \\
20 & 5.67 & 7.57 & 1.20 & 1.89 & 0.0118 & 0.500 & 0.272 & 0.772 \\
40 & 7.50 & 7.85 & 2.74 & 2.23 & 0.0103 & 0.526 & 0.237 & 0.763 \\
40 & 7.44 & 7.50 & 2.74 & 1.93 & 0.0122 & 0.527 & 0.281 & 0.808 \\
60 & 9.68 & 8.18 & 4.80 & $(2.83)$ & 0.0085 & 0.531 & 0.196 & 0.727 \\
60 & 9.13 & 7.77 & 4.80 & 1.83 & 0.0107 & 0.484 & 0.247 & 0.731
\end{tabular}

Note. -Thiosulphate solution $0.04 N$ was used in the measurements of this table to avoid a too great change in volume. 


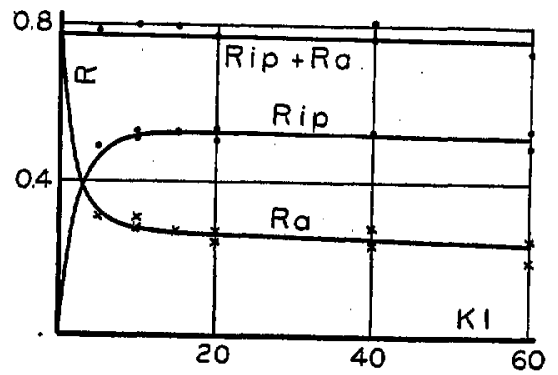

Fig. 4 (Cf. Table IX)

TABLE X

$t=9.5$

\begin{tabular}{|c|c|c|c|c|c|}
\hline As & 10.00 & 20.00 & 10.00 & 10.00 & 10.00 \\
\hline$K I$ & 245 & 245 & 490 & 245 & 245 \\
\hline$C r$ & 20 & 20 & 20 & 40 & 20 \\
\hline$A c$ & 242 & 242 & 242 & 242 & 484 \\
\hline$h$ & 2.00 & 3.60 & 2.60 & 3.60 & 6.00 \\
\hline$A s-x^{\prime}$ & 9.15 & 18.30 & 9.18 & 8.50 & 7.70 \\
\hline$x^{\prime}$ & 0.85 & 1.70 & 0.82 & 1.50 & 2.30 \\
\hline & 0.35 & 0.35 & 0.88 & 0.68 & I. 40 \\
\hline $\begin{aligned} h-i c & \\
& h-i c\end{aligned}$ & I. 65 & 3.25 & 1.72 & 2.92 & 4.60 \\
\hline$r=\frac{n}{x^{\prime}}$ & I.94 & $1.9 \mathrm{I}$ & 2.IO & 1.95 & 2.00 \\
\hline$k^{\prime}$ & 0.0040 & 0.0040 & 0.0037 & 0.0074 & 0.0119 \\
\hline & $0.0 \mathrm{I} 14$ & 0.0116 & 0.0114 & 0.0217 & 0.0318 \\
\hline$r=\frac{k-k^{\prime}}{k}$ & 1.9 & I.9 & $2 . I$ & 2.0 & 1.7 \\
\hline
\end{tabular}

\section{TABLE XI}

$A s, 5.00 ; K I, 196 ; C r, 20 ; A C, 484$

\begin{tabular}{|c|c|c|c|c|c|c|c|}
\hline$t$ & $h$ & ic (calc.) & $h-i c($ calc. $)$ & $t$ & $h$ & $i c(c a l c)$ & $h-i c(c a l c)$. \\
\hline 3 & 1.20 & 0.35 & 0.85 & 55 & I 3.92 & 5.85 & 8.07 \\
\hline 5 & I.92 & 0.58 & I. 34 & 60 & 14.64 & 6.38 & 8.26 \\
\hline 9 & 3.36 & I.04 & 2.32 & 65 & $\mathrm{I} 5.28$ & 6.90 & 8.38 \\
\hline IO & 3.80 & I. I 5 & 2.65 & 70 & I 5.96 & $7 \cdot 40$ & 8.56 \\
\hline I 5 & $5 \cdot 40$ & I. 72 & 3.68 & 75 & I 6.56 & 7.90 & 8.66 \\
\hline 20 & 6.72 & 2.28 & $4 \cdot 44$ & 80 & 17.24 & 8.40 & 8.84 \\
\hline 25 & 8.00 & 2.82 & 5.18 & 85 & 17.92 & 8.90 & 9.02 \\
\hline 30 & 9.20 & $3 \cdot 32$ & $5 \cdot 9^{8}$ & 95 & 19. 16 & $9 \cdot 90$ & 9.26 \\
\hline 35 & 10.32 & $3.8 \mathrm{I}$ & $6.5 \mathrm{I}$ & 100 & 19.76 & 10.40 & $9 \cdot 36$ \\
\hline 40 & I I. 28 & $4 \cdot 30$ & 6.98 & IO5 & 21.16 & 10.88 & 9.28 \\
\hline 45 & 12.32 & 4.82 & 7.50 & I 40 & 23.68 & 14.20 & $9.4^{8}$ \\
\hline 50 & I 3.16 & $5 \cdot 34$ & 7.82 & 160 & 25.28 & I 5.80 & 9.48 \\
\hline
\end{tabular}

Note. - In making the iodine titrations 0.04 thiosulphate was used to avoid a too great change in volume. 
Fig. 5 is plotted from the results of Table XI together with the values of $x$ from Table XII of my paper on the oxidation of arsenious acid, and the values of $x^{\prime}$ from Table XVIII of the present paper.

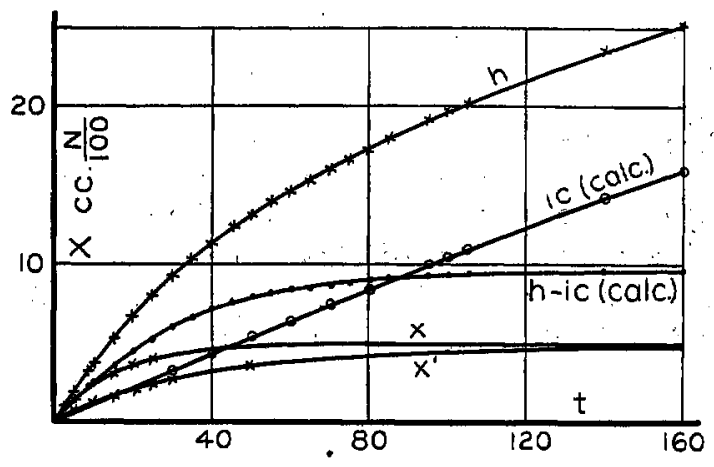

Fig. 5 (Cf. Table XI)

TABLE XII

$A s, 9.94 ; K I, 98 ; C r, 20 ; A c, 242$

\begin{tabular}{|c|c|c|c|c|c|c|c|}
\hline$t$ & $h$ & $A s-x^{\prime}$ & $x^{\prime}$ & $i c$ & $h-i c$ & $r=\frac{h-i c}{x^{\prime}}$ & $k^{\prime}$ \\
\hline IO & I. 74 & 9.00 & 0.94 & 0.10 & r.64. & I.75 & 0.0043 \\
\hline IO & I. 93 & 9.16 & 0.78 & 0.10 & I. 88 & $(2.4 \mathrm{I})$ & $(0.0036)$ \\
\hline I 5 & 2.52 & 8.56 & I. 38 & 0.15 & 2.37 & 1.72 & 0.0043 \\
\hline 20 & 3.40 & 8.23 & $1.7 \mathrm{I}$ & 0.20 & 3.20 & 1.87 & $0.004 \mathrm{I}$ \\
\hline 30 & 4.75 & $7 \cdot 52$ & 2.42 & 0.30 & 4.45 & 1.84 & 0.0040 \\
\hline
\end{tabular}

Avg. 1.80 Avg.0.004I

TABLE XIII

As, 9.94; KI, 196; $C r, 20 ; A c, 242$

\begin{tabular}{r|c|c|c|c|c|c|c|}
$r t$ & $h$ & $A s-x^{\prime}$ & $x^{\prime}$ & $i c$ & $h-i c$ & $r=\frac{h-i c}{x^{\prime}}$ & $k^{\prime}$ \\
\hline 5 & 0.98 & 9.48 & 0.46 & 0.14 & 0.84 & $\mathrm{I} .83$ & $0.004 \mathrm{I}$ \\
10 & 1.88 & 9.00 & 0.94 & 0.27 & $\mathrm{I} .6 \mathrm{I}$ & $\mathrm{I} .7 \mathrm{I}$ & 0.0043 \\
$\mathrm{I} 5$ & 2.78 & 8.63 & $1.3 \mathrm{I}$ & $0.4 \mathrm{O}$ & 2.38 & $\mathrm{I} .83$ & $0.004 \mathrm{I}$ \\
$\mathrm{I} 5$ & 2.74 & 8.55 & $\mathrm{I} .39$ & 0.40 & 2.34 & $\mathrm{I} .69$ & 0.0044 \\
20 & 3.66 & 8.23 & $\mathrm{I} .7 \mathrm{I}$ & 0.54 & 3.12 & 1.82 & $0.004 \mathrm{I}$ \\
25 & 4.40 & 7.84 & 2.10 & 0.67 & 3.73 & 1.78 & $0.004 \mathrm{I}$ \\
30 & 5.14 & 7.58 & 2.36 & $0.8 \mathrm{I}$ & 4.33 & 1.84 & 0.0039
\end{tabular}

Avg. 1.80 Avg.0.004I 
TABLE XIV

$A s, 9.94 ; K I, 294 ; C r, 20 ; A c, 242$

\begin{tabular}{r|c|c|c|c|c|c|c|c}
\hline \hline$t$ & $h$ & $A s-x^{\prime}$ & $i c$ & $r=\frac{h-i c}{x^{\prime}}$ & $k^{\prime}$ & $x$ & $x$ (calc) & $h-i c+x^{\prime}$ \\
& & & & & & & \\
IO & 2.00 & 9.18 & 0.43 & 2.07 & 0.0035 & - & - & - \\
I0 & 2.17 & 9.12 & 0.43 & 2.12 & 0.0037 & 2.34 & 2.56 & 2.56 \\
I 5 & 3.15 & 8.67 & 0.64 & 1.98 & 0.0040 & 3.28 & 3.75 & 3.78 \\
I5 & 3.11 & 8.74 & 0.64 & 2.08 & 0.0037 & 3.28 & 3.75 & 3.67 \\
20 & 4.00 & 8.42 & 0.85 & 2.07 & 0.0036 & 3.99 & 4.76 & 4.67 \\
30 & 5.67 & 7.74 & 1.28 & 2.00 & 0.0036 & 5.35 & 6.68 & 6.59 \\
40 & 7.26 & 7.12 & I.70 & 1.97 & 0.0038 & 6.50 & 8.86 & 8.38
\end{tabular}

Note.-The values of $x$ of this table are taken from Table VIII of the paper on the oxidation of arsenious acid; $x$ (calc.) is calculated as described in the foot-note on page 73 .

TABLE XV

$A s, 5.00 ; K I, 196 ; C r, 20 ; A c, 242$,

\begin{tabular}{c|c|c|c|c|c|c|c}
\hline$t$ & $h$ & $A s-x^{\prime}$ & $x^{\prime}$ & $i c$ & $h-i c$ & $r=\frac{h}{x^{\prime}}$ & $k^{\prime}$ \\
\hline IO & 1.15 & 4.57 & 0.43 & 0.27 & 0.88 & 2.05 & 0.0039 \\
I5 & 1.62 & 4.40 & 0.60 & 0.40 & 1.22 & 2.03 & 0.0037 \\
20 & 2.10 & 4.19 & 0.81 & 0.54 & 1.56 & 1.93 & 0.0038 \\
25 & 2.58 & 4.04 & 0.96 & 0.67 & 1.91 & 1.99 & 0.0037 \\
30 & 3.04 & 3.85 & 1.I5 & $0.8 \mathrm{I}$ & 2.23 & 1.94 & 0.0038
\end{tabular}

TABLE XVI

As, 5.00: $K I$, i $96 ; C r, 40 ; A c, 242$

\begin{tabular}{|c|c|c|c|c|c|c|c|}
\hline$t$ & $h$ & $A s-x^{\prime}$ & $x^{\prime}$ & ic & $h-i c$ & $r=\frac{h-i c}{x^{\prime}}$ & $k^{\prime}$ \\
\hline 5 & I. IO & 4.69 & $0.3 \mathrm{I}$ & 0.24 & 0.86 & $(2.77)$ & $(0.0056)$ \\
\hline I0 & 2.02 & 4.24 & 0.76 & 0.48 & I. 54 & 2.03 & 0.0072 \\
\hline I 5 & 2.83 & 3.85 & I. 15 & 0.72 & 2. I I & I. 94 & 0.0076 \\
\hline 20 & 3.63 & $3.5^{8}$ & I. 42 & 0.96 & 2.67 & 1.88 & 0.0073 \\
\hline $30^{\circ}$ & 5.09 & 3.05 & I.95 & I. 44 & 3.65 & 1.87 & 0.0072 \\
\hline
\end{tabular}

TABLE XVII Avg. 1.93 Avg. 0.0073

As, 9.94; KI, 196;Cr, 40;Ac, 242

\begin{tabular}{|c|c|c|c|c|c|c|c|}
\hline$t$ & $h$ & $A s-x^{\prime}$ & $x^{\prime}$ & ic & $h-i c$ & $r=\frac{h-i c}{x^{\prime}}$ & $k^{\prime}$ \\
\hline 5 & I. 84 & 9.14 & 0.80 & 0.25 & I. 59 & I.99 & 0.0073 \\
\hline IO & 3.46 & 8.44 & $\mathrm{r} .50$ & 0.50 & 2.96 & 1.97 & $0.007 \mathrm{I}$ \\
\hline I 5 & 4.87 & 7.79 & 2.1 5 & 0.75 & $4 \cdot 12$ & I.92 & $0.007 \mathrm{I}$ \\
\hline
\end{tabular}


The Induction by Arsenious Acid, Etc.

TABLE XVIII

$A s, 5.00 ; K I$, I96; Cr, 20; $A c, 484$

\begin{tabular}{|c|c|c|c|c|c|c|c|}
\hline$t$ & $h$ & $A s-x^{\prime}$ & $x^{\prime}$ & ic & $h-i c$ & $r=\frac{h-i c}{x^{\prime}}$ & $k^{\prime}$ \\
\hline 5 & I.90 & 4.30 & 0.70 & 0.60 & I. 30 & I. 86 & $(0.0131)$ \\
\hline IO & 3.59 & 3.80 & 1.20 & $\mathrm{I} .20$ & 2.39 & 1.99 & 0.0119 \\
\hline I 5 & 5.16 & 3.37 & I. 63 & I. 80 & 3.36 & 2.06 & 0.0114 \\
\hline 20 & 6.48 & 2.95 & 2.05 & 2.40 & 4.08 & 1.99 & 0.0115 \\
\hline
\end{tabular}

TABLE XIX

$A s, 9.94 ; K l$, I96; $C r, 20 ; A c, 484$

\begin{tabular}{|c|c|c|c|c|c|c|}
\hline$k$ & $A s-x^{\prime}$ & $x^{\prime}$ & $i \dot{c}$ & $h-i c$ & $r=\frac{h-i c}{x^{\prime}}$ & $k^{\prime}$ \\
\hline 3.05 & $8.6 I$ & I.33 & 0.60 & 2.45 & I. 84 & (0.0125) \\
\hline 5.67 & 7.57 & 2.37 & 1.20 & $4 \cdot 47$ & I. 89 & 0.0118 \\
\hline 8.03 & 6.64 & 3.30 & I. 80 & 6.25 & vg. $1.87 \mathrm{Ar}$ & $\begin{array}{r}0.0117 \\
0.0118\end{array}$ \\
\hline
\end{tabular}

\section{TABLE XX}

As, 9.94; KI, I96; Cr, 20;AC, 242 (Temperature $10^{\circ} \mathrm{C}$ )

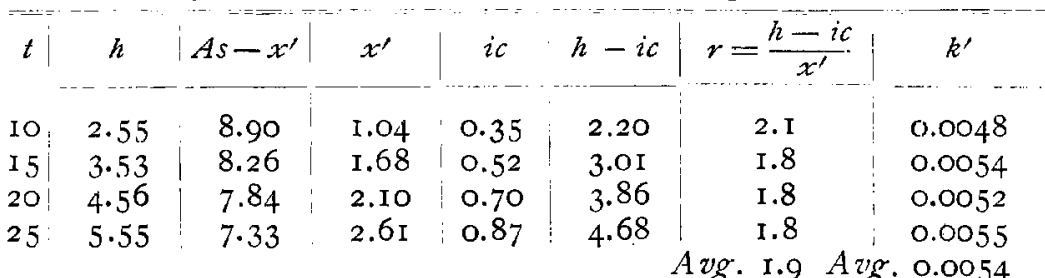

\section{TABLE XXI}

$K I, 98 ; \mathrm{Cr}, 20$;

$A c, 242 \quad K I$, 196; Cr, 20; $A c, 242 \quad K I, 294 ; C r, 20 ; A c, 242$.

\begin{tabular}{|c|c|c|c|c|c|c|c|c|c|}
\hline$i c$ & $t$ & $\begin{array}{l}i c \\
0^{i c} \mathrm{C}\end{array}$ & $\mathrm{O}^{0^{t} \mathrm{C}}$ & $\begin{array}{l}i c \\
10^{\circ} \mathrm{C}\end{array}$ & ${ }_{10}^{t} 0^{\circ} \mathrm{C}$ & ic & $t$ & $i c$ & $t$ \\
\hline 0.20 & I I & 0.25 & 9.2 & 0.30 & IO & 0.48 & I I & 2.00 & $4^{6}$ \\
\hline 0.2 & 30 & 0.50 & I 8.7 & 0.50 & 15 & $0.8 \mathrm{I}$ & I9 & 2.40 & 57 \\
\hline 0.8 & 86 & I.IO & 42 & 0.85 & 25 & 1.40 & $3 \mathrm{I}$ & 3.20 & 75 \\
\hline I. 23 & I 20 & 1.20 & 45.5 & I.IO & 30 & 1.48 & 35 & 3.84 & 90 \\
\hline
\end{tabular}


$K I, 392 ; C r, 20 ; A C, 242 . K I, 245 ; C r, 20 ; A C, 242 . K I, 686 ; C r, 20 ; A c, 242$

\begin{tabular}{|c|c|c|c|c|c|c|c|c|c|c|c|}
\hline$i c$ & $t$ & $i c$ & $t$ & $i c$ & $t$ & ic & $t$ & $i c$ & $t$ & $i c$ & $t$ \\
\hline 0.24 & 4.2 & 0.87 & 14.5 & $0.3 \mathrm{I}$ & 9.5 & I.33! & 38 & 0.40 & 2.5 & 2.00 & I 3.5 \\
\hline 0.30 & 5.0 & 1.00 & 17.0 & 0.50 & 14.0 & 1.68 & 47.5 & 0.80 & 5.5 & 3.55 & 26 \\
\hline 0.58 & 10.0 & I. $2 \mathrm{I}$ & 20.2 & 0.67 & 19.0 & 2.00 & 57 & I. IO & 7.5 & 3.75 & 27 \\
\hline 0.80 & I 3.2 & $\mathrm{I} .20$ & 20.7 & I.02 & 28.5 & 2.64 & 76 & I. 45 & $10^{\circ}$ & 4. Io & 30.4 \\
\hline
\end{tabular}

$K I$, I96; $C r, 40 ; A c, 242 . K I$, I 96; $C r, 20 ; A c, 484 . K I, 490 ; C r$, I0; $A c, 242$

\begin{tabular}{c|c|c|c|c|c}
\hline$i c$ & $t$ & $i c$ & $t$ & $i c$ & $t$ \\
\hline 0.27 & 5 & 0.60 & 5 & 0.29 & 6 \\
0.44 & 7.5 & 1.20 & 10 & 0.52 & 13 \\
0.57 & 10 & 1.82 & 15 & 0.66 & 17 \\
0.78 & 15 & 2.43 & 20 & 0.76 & 19.5 \\
1.03 & 20 & 3.00 & 25 & 0.95 & 25.5 \\
1.75 & 35 & 3.60 & 31 & 1.19 & 32 \\
1.98 & 40 & 4.20 & 35 & 1.42 & 40 \\
2.40 & 50 & - & - & - & -
\end{tabular}

TABLE XXII

\begin{tabular}{|c|c|c|c|c|c|c|c|}
\hline$\underset{r^{\prime}}{\text { Table }}$ & $\underset{r^{\prime}}{\text { Table }}$ & $\begin{array}{c}\text { Table } \\
\text { XIV } \\
r^{\prime}\end{array}$ & $\begin{array}{c}\text { Table } \\
\mathrm{XV} \\
r^{\prime}\end{array}$ & $\begin{array}{c}\text { Table } \\
\text { XVI } \\
r^{\prime}\end{array}$ & $\begin{array}{l}\text { Table } \\
\text { XVII } \\
r^{\prime}\end{array}$ & $\begin{array}{l}\text { Table } \\
\text { XVIII } \\
r^{\prime}\end{array}$ & $\underset{r^{\prime}}{\text { Table }}$ \\
\hline I. .7 & I. 7 & I. 5 & $(2.6)$ & $(2.8)$. & I. 7 & I.4 & 2.4 \\
\hline$(2.5)$ & 1. 6 & 2.0 & I. 7 & 2.0 & I. 4 & 2.3 & I. 4 \\
\hline I.5 & I. 5 & I. 7 & I. 5 & I. 5 & - & I.5 & - \\
\hline I. 6 & I. 5 & I. 7 & - & - & - & - & - \\
\hline - & I. 5 & I. 5 & - & - & - & - & 一 \\
\hline$r^{\prime \prime}$ & $r^{\prime \prime}$ & $r^{\prime \prime}$ & $\dot{r}^{\prime \prime}$ & $r^{\prime \prime}$ & $r^{\prime \prime}$ & $r^{\prime \prime}$ & $r^{\prime \prime}$ \\
\hline I. 9 & I.9 & I.9 & $(2.5)$ & $(2.7)$ & r.9 & $(3.5)$ & 2.3 \\
\hline 2.1 & I. 8 & $2 . I$ & I.9 & 2.2 & 1.8 & $(3.0)$ & 2.I \\
\hline I. 8 & I. 8 & 2.I & 2.0 & 2.I & I. 6 & 2.4 & I.9 \\
\hline I.9 & I. 8 & 2.0 & 1.8 & 1.9 & - & 2.3 & - \\
\hline 1.7 & I. 8 & 1.9 & 1.7 & I. 8 & - & - & $T$ \\
\hline & I. 7. & 1.8 & & - & - & - & - \\
\hline - & I 6 & I. 6 & - & - & 一 & 一 & - \\
\hline
\end{tabular}


TABLE XXIII

\begin{tabular}{|c|c|c|c|c|c|c|c|c|}
\hline Table & IV & \multicolumn{2}{|r|}{$\mathrm{V}$} & VI & \multicolumn{2}{|c|}{ VII } & VII & IX \\
\hline As & IO & \multicolumn{2}{|c|}{ IO } & IO & \multicolumn{2}{|c|}{20} & IO & IO \\
\hline$K I$ & $300-$ & \multicolumn{2}{|c|}{$150+$} & $150+$ & \multicolumn{2}{|c|}{$300+$} & $150+$ & $\mathrm{I} 5 \mathrm{O}^{-}$ \\
\hline$C r$ & 20 & \multicolumn{2}{|c|}{20} & 20 & \multicolumn{2}{|c|}{20} & 40 & 20 \\
\hline$A c$ & I $2 \mathrm{I}$ & \multicolumn{2}{|c|}{242} & 242 & \multicolumn{2}{|c|}{242} & 242 & 484 \\
\hline$k$ & 0.004 & \multicolumn{2}{|c|}{$0.0 \mathrm{II} 4$} & $0.011_{4}$ & \multicolumn{2}{|c|}{0.0116} & 0.0217 & 0.0318 \\
\hline$k^{\prime}$ & 0.001 & \multicolumn{2}{|c|}{0.0037} & 0.0038 & \multicolumn{2}{|c|}{0.0037} & 0.0076 & 0.0112 \\
\hline$r+\mathrm{I}=\frac{k}{k^{\prime}}$ & $3 \cdot 4$ & \multicolumn{2}{|r|}{3.1} & 3.0 & \multicolumn{2}{|c|}{3.1} & 2.9 & 2.9 \\
\hline$r=\frac{k-k^{\prime}}{k^{\prime}}$ & 2.4 & \multicolumn{2}{|r|}{$2 . I$} & 2.0 & \multicolumn{2}{|c|}{$2 . I$} & I.9 & 1.9 \\
\hline Table & \multicolumn{2}{|r|}{ XIII } & $\mathrm{XIV}$ & $\mathrm{xV}$ & $X V I$ & XVII & XVIII & XIX \\
\hline As & IO & IO & 10 & 5 & 5 & IO & 5 & IO \\
\hline$K I$ & 98 & I96 & 294 & 196 & I96 & 196 & 196 & 196 \\
\hline $\mathrm{Cr}$ & 20 & 20 & 20 & 20 & 40 & 40 & 20 & 20 \\
\hline Ac & 242 & 242 & 242 & 242 & 242 & 242 & 484 & 484 \\
\hline$k$ & O.OI I 4 & $0.0 I_{4}$ & 0.0114 & $0.01 I_{4}$ & 0.0200 & 0.0217 & 70.0300 & 0.0318 \\
\hline$k^{\prime}$ & $0.004 \mathrm{I}$ & $0.004 \mathrm{I}$ & 0.0037 & 0.0038 & 0.0073 & 0.0072 & 20.0I I 6 & 0.0118 \\
\hline$r+\mathrm{I}=\frac{k}{k^{\prime}}$ & 2.8 & 2.8 & $3 . I$ & 3.0 & 3.0 & 3.0 & 2.6 & 2.7 \\
\hline$r=\frac{k-k^{\prime}}{k^{\prime}}$ & I. 8 & I. 8 & $2 . I$ & 2.0 & 2.0 & 2.0 & I. 6 & I. 7 \\
\hline
\end{tabular}

\section{Discussion of the Experimental Data}

The measurements recorded above together with those of my previous papers on the action of chromic acid on iodide ${ }^{1}$ and arsenious acid ${ }^{2}$ constitute all the experimental data on which the theory developed in the following pages is based. In order to collect all the facts together the generalizations deduced in the last-mentioned papers are quoted in the following paragraphs along with the general conclusions drawn from the tables of the present paper.

\section{Effect of the Concentration of the Iodide}

Tables IV-IX (in each of which $t$ is constant) show that increasing the concentration of the iodide causes a gradual

2 See equation (2). 
increase in the value of $h-i c$ (the iodine liberated due to the presence of the arsenious acid), and a gradual decrease in the value of $x^{\prime}$ (the arsenious acid oxidized in the presence of iodide), a maximum value of $h-i c$ and a minimum value of $x^{\prime}$ being reached when the concentration of the iodide is about fifteen times that of the arsenious acid, that is, when $K I=\mathrm{I}_{5} A \mathrm{~s}$. Further increase in the concentration of the iodide does not appreciably affect these limiting values of $h-i c$ and $x^{\prime}$.

When the iodide is in excess the value of the ratio, $\frac{h-i c}{x^{\prime}}$, is 2.00 within the limit of the experimental error, no matter what the concentrations of the other reagents are. Thus in Tables XII-XX the average values of this ratio are, I.80, I.80, 2.04, I.99, I.93, I.95, I.98, I.87 and I.9 (at 10 ${ }^{\circ} \mathrm{C}$ ); in Table IV the values are, 2.06, 2.25, 2.07, 2.18, 2.04 and $I .94$; and in Table X, I.94, I.9I, 2.I0, I.95 and 2.00. Additional evidence is afforded by the measurements of Tables V-IX (Figs. I-4) and the curves of Fig. 5. ${ }^{1}$ The slight deviations in both directions, from the number 2.00 must be ascribed to experimental error, for the ratio in question is the quotient of two small numbers, each of which is the difference between two small readings of the burette, and owing to the nature of the method of analysis it usually happens that when one of these numbers (say $h-i c$ ) is too small the other $\left(x^{\prime}\right)$ is too large.

Tables V-IX and the curves of Figs. I-4, show that addition of potassium iodide lessens the rate of oxidation of arsenious acid by chromic acid, the retardation increasing with the concentration of the iodide up to a certain point from which on ( $R a$, when $K I=o$, being equal to $R a c)$,

$$
R a,(\text { KI excess })=\frac{I}{3} \cdot \text { Rac. }
$$

Additional evidence of relation (7) is afforded by Table XXIII in which $k$ is seen to closely equal $3 k^{\prime}$.

1 The curve, $h-i c$, approaches the line, $X=I O=2 A s$, asymptotically, and simultaneously the curve, $x^{\prime}$, approaches the line, $X=5=A s$. 
Tables V-IX and Figs. I-4 further show that the rate at which iodine is liberated in solutions containing arsenious and chromic acids, after correcting for the direct oxidation of iodide by chromic acid, increases with the concentration of the iodide up to a certain point from which on,

$$
\begin{aligned}
\text { Rip },(K I \text { excess }) & =2 R a,(K I \text { excess }) \\
& =\frac{2}{3} \cdot \text { Rac. }
\end{aligned}
$$

The last columins of Tables V-IX and the upper curves of Figs. I-4, show that the sum, $R a+R i p$, that is, the rate of reduction of chronic acid ${ }^{1}$ after correcting for the direct action of the chromic acid on the iodide, is the same for all concentrations of iodide from zero up, or,

$$
R a+R i-R i c=R a c
$$

that is,

$$
R a+R i p=R a c .
$$

Thus $M$ of equation (5) is - Rip.

In the absence of arsenious acid the rate of oxidation of iodide by chromic acid is connected with the concentration of the iodide by a quadratic relation (see equation I).

1 On comparing the above Tables of results with those on the oxidation of arsenious acid in the absence of iodide, it will be noticed, however, that for any fixed interval of time the total oxidation is greater in the presence than in the absence of the iodide, that is, $h-i c+x^{\prime}$ is greater than $x$ for a fixed time. This is due to the fact that in the former case the concentration of the arsenious acid (on which the $h-i c$ reading depends) does not fall off so rapidly as in the latter case. If the average concentration of the arsenious acid had remained the same as it did in the experinlents in which iodide was present, we would have for the value of $x$ :

$$
x\left(c a^{\prime} c\right)=x . \frac{A s . t-\int_{0}^{t} x^{\prime \prime} \cdot d t}{A s . t-\int_{0}^{t} x \cdot d t} ;
$$

and as shown by Table XIV, $x($ calc $)$ is equal to the experimentally determined value, $h-i c+x^{\prime}$. For the method of computation, see Jour. Phys. Chem., ro, 423 (1906). 


\section{Effect of the Concentration of the Arsenious Acid}

In the absence of iodide the rate of oxidation of arsenious acid by chromic acid is proportional to the first power of the concentration of the arsenious acid. This suggested computing the constant of the "first order" in the tables of the present paper. The constancy of $k^{\prime}$ in each of the Tables XII-XX, and more particularly the fact that changing the concentration of the arsenious acid in these tables as well as in Tables V, VI and VII, does not effect the value of $k^{\prime}$, shows that in the presence as well as in the absence of iodide the rate of oxidation of arsenious acid by chromic acid is proportional to the first power of the concentration of the arsenious acid.

Since in the presence of excess of iodide, $h-i c=2 x^{\prime}$. (see page 72 ), the rate $R i p$ is twice the rate $R a$, (KIexcess), and is therefore, like the latter, proportional to the concentration of the arsenious acid. The fact that $R i p+R a=R a c$ for all concentrations of iodide from zeru up, is also proof that Rip is proportional to the first power of the concentration of the arsenious acid.

\section{Effect of the Concentration of the Bichromate}

Comparison of Tables V and VIII, XV and XVI, XIII and XVII, shows that doubling the concentration of the bichromate almost doubles the value of $k^{\prime}$, that is, almost doubles the rate of oxidation of arsenious acid, $R a$, when iodide is present; or the rate $R a$ is proportional to about the o.9th power of the concentration of the bichromate.

In the absence of iodide the same relation was found to hold; that is, Rac is proportional to the 0.9 th power of the concentration of the bichromate.

Since in the presence of excess of iodide $h-i c=2 x^{\prime}$, the rate $R i p$ is twice the rate $R a,(K I$ excess), and is therefore like the latter proportional to the same (o.gth) power of the concentration of the bichromate; and the fact that Rip+ $R a=R a c$ is also proof that the rate $R i p$ is proportional to the 0.9 th power of the concentration of the bichromate.

In the absence of arsenious acid the rate of oxidation of 
iodide (Ric) was found to be proportional to a power of the bichromate slightly less than the first (except when very dilute solutions of bichromate were employed, when the power was the first exactly). In the above tables ic is scarcely doubled for a fixed interval by doubling the concentration of the bichromate.

Thus the rates $R a c R a, R i p$ and $R i c$ are each proportional to a power of the concentration of the bichromate slightly less than the first.

\section{Effect of the Concentration of the Sulphuric Acid}

Doubling the concentration of the sulphuric acid nearly trebles the rates $R a$ and $R i p$ in Tables $V$ and IX, and the constants $k^{\prime}$ in Tables XV and XVIII, XIII and XIX. Thus the rates $R a$ and $R i p$ are proportional to the 1.4 th power of the concentration of the sulphuric acid.

The rate of oxidation of arsenious acid in the absence of iodide is also proportional to the I.4th power of the concentration of the sulphuric acid. This unusual fractional power is thus common to the kinetic formulae for the rates $R a c, R a$ and $R i p$, while the rate of oxidation of iodide in the absence of arsenious acid (Ric) is proportional to the square of the concentration of the sulphuric acid.

\section{Effect of the Temperature}

Changing the temperature from $0^{\circ} \mathrm{C}$ to $10^{\circ} \mathrm{C}$ causes a change in the constant $k^{\prime}$ of 29 percent (0.004 1 to 0.0053 , Tables XIII and XX). Since in these tables $h-i c=2 x^{\prime}$, the rate $R i p$ is also increased by the same amount.

Changing the temperature has practically the same effect on the rate of oxidation of arsenious acid in the absence of iodide $(R a c)$; the experiments showed that changing the temperature from $0^{\circ} \mathrm{C}$ to $10^{\circ} \mathrm{C}$ causes a change of 26 percent in the constant $k$ (O.OII4 to o.or44).

Thus the rates $R a c, R a$ and $R i p$ have the same unusually low temperature coefficient, viz., less than I.3.

The temperature coefficient of the rate of oxidation of iodide by chromic acid is also very low, viz., I.4. 


\section{Short Statement of the Results}

The following are the general results of the experiments:

$$
\begin{aligned}
& R a c=k \cdot A s(C r)^{\circ 9} \cdot(A c)^{r \cdot 4} \\
& R a=k^{\prime} \cdot A s(C r)^{0.9} \cdot(A c)^{\mathrm{I} .4} \\
& R i c=K \cdot\left(m \cdot K I+n \cdot(K I)^{2}\right) \cdot C r \cdot(A c)^{2} \text {, } \\
& R i-R i c=R i p=k^{\prime \prime} \cdot A s \cdot(C r)^{0.9} \cdot(A c)^{\mathrm{r} .4} \\
& \text { Compare equation (I) } \\
& \text { and } \quad R a c=R a+R i p \text {. }
\end{aligned}
$$

The constants $k^{\prime}$ and $k^{\prime \prime}$ depend on the amount of iodide present: as the concentration of the iodide increases, $k^{\prime}$ decreases from $k$, for $K I=0$, to $\frac{k}{3}$, for $K I$, excess; and simultaneously $k^{\prime \prime}$ increases from o to $2 k^{\prime}$ or $2 \frac{k}{3}$.

When the iodide is in excess the ratio $\frac{h-i c}{x^{\prime}}$ is equal to 2.

The rates $R a c, R a$, (KI excess) and Rip, (KI excess) have the same unusually low temperature coefficient, viz., less than I.3 (from $0^{\circ} C$ to $10^{\circ} C$ ); the temperature coefficient for the rate $R i c$ is about I.4.

\section{THEORETICAL PART}

The above results show that:

(a) The rate of reduction of chromic acid in solutions containing arsenious acid and iodide is equal to the sum of the rates of reduction of chromic acid by arsenious acid and by iodide taken separately, or, in other words, the acceleration of the rate of oxidation of iodide is just equal to the retardation in the rate of oxidation of arsenious acid.

(b) The effects of temperature and the concentrations of the various reagents are the same for the three rates:

(i) Oxidation of arsenious acid by chromic acid in the absence of jodide (Rac).

(ii) Oxidation of arsenious acid by chromic acid in the presence of excess of iodide ( $R a, K I$ excess).

(iii) Oxidation of iodide in the presence of arsenious acid, when the iodide is in excess (Rip, KI excess). 
(c) The ratio $R a: R i p$ is independent of the concentrations of the bichromate and sulphuric acid for all concentrations of iodide.

(d) In the absence of arsenious acid the rate of oxidation of iodide is proportional to powers of the concentrations of the reagents somewhat different from those of rates $(i)$, (ii) and (iii) of $(b)$.

These facts suggest the theory of the formation of a primary oxide when chromic acid acts on arsenious acid, and the succeeding paragraphs will show that the above experimental results may be satisfactorily accounted for by the following assumptions.

\section{Assumptions}

(a) That arsenious acid acting on chromic acid forms slowly a primary oxide.

(b) That this oxide is completely and instantaneously reduced by either arsenious acid or iodide.

(c) That the fractions of the primary oxide reduced by arsenious acid and iodide respectively, in solutions containing both, depend on their relative concentrations; when the iodide is in large excess the amount of the primary oxide reduced by the arsenious acid is negligible.

The primary oxide may be one of the following: 1

(I) A peroxide of arsenic represented by the formula, $\mathrm{As}_{2} \mathrm{O}_{0}$, or one of its hydrates, e. g., $\mathrm{H}_{3} \mathrm{AsO} \mathrm{O}_{6}$, the reactions being as follows:

(i) Slow formation of the peroxide, $\mathrm{As}_{2} \mathrm{O}_{3}+2 \mathrm{~K}_{2} \mathrm{Cr}_{2} \mathrm{O}_{7}+{ }_{3} \mathrm{H}_{2} \mathrm{SO}_{4}=\mathrm{As}_{2} \mathrm{O}_{9}+2 \mathrm{Cr}_{2}\left(\mathrm{SO}_{4}\right)_{3}+2 \mathrm{~K}_{2} \mathrm{SO}_{4}+3 \mathrm{H}_{2} \mathrm{O}$; or,

$$
\text { - } \overline{A s} \overline{O_{3}}+\bar{C} r_{2} \mathrm{O}_{7}+2 \stackrel{+}{H}=-\overline{A s} \bar{O}_{6}+\mathrm{Cr}_{2} \mathrm{O}_{3}+\mathrm{H}_{2} \mathrm{O} .
$$

(ii) Rapid reduction by arsenious acid or iodide, $A s_{2} O_{3}+2 A s_{2} O_{3}=3 A s_{2} O_{5} ;$ or, $\bar{A} \bar{s} \bar{O}_{6}+\overline{2} \bar{A} \overline{S O}_{3}=\overline{3} \overline{A s} \bar{O}_{4}$ and

1 For methods of determining the degree of oxidation of the primary oxide, see p. 83 . 


$$
\begin{aligned}
& A s_{2} \mathrm{O}_{9}+8 H I=A s_{2} \mathrm{O}_{3}+{ }_{4} I_{2}+{ }_{4} \mathrm{H}_{2} \mathrm{O} ; \text { or, } \\
& \qquad \overline{A s O_{6}}+4 \bar{I}+4 \bar{H}=\overline{A s O_{4}}+2 I_{2}+2 H_{2} \mathrm{O} .
\end{aligned}
$$

(2) An intermediate oxide of chromium of the formula $\mathrm{Cr}_{2} \mathrm{O}_{5}$, or one of its hydrates, e.g., $\mathrm{H}_{2} \mathrm{Cr}_{2} \mathrm{O}_{\mathrm{e}}$, the reactions being as follows:

(i) Slow formation of the intermediate oxide (peroxide), $A s_{2} \mathrm{O}_{3}+2 \mathrm{~K}_{2} \mathrm{Cr}_{2} \mathrm{O}_{7}=\mathrm{As}_{2} \mathrm{O}_{5}+2 \mathrm{~K}_{2} \mathrm{Cr}_{2} \mathrm{O}_{8} ;$ i. e., $\mathrm{As}_{2} \mathrm{O}_{3}+{ }_{4} \mathrm{CrO}_{3}=\mathrm{As}_{2} \mathrm{O}_{5}+2 \mathrm{Cr}_{2} \mathrm{O}_{5}$

or,

$$
\overline{A s} \bar{O}_{3}+\overline{C r} \bar{r}_{2} \bar{O}_{7}=\overline{A s} \bar{O}_{4}+\bar{C} \bar{r}_{2} O_{6} .
$$

(ii) Rapid reduction by arsenious acid or iodide, $\mathrm{Cr}_{2} \mathrm{O}_{5}+\mathrm{As}_{2} \mathrm{O}_{3}=\mathrm{Cr}_{2} \mathrm{O}_{3}+\mathrm{As}_{2} \mathrm{O}_{5}$; or,

$$
\overline{\mathrm{C}} \bar{r}_{2} \mathrm{O}_{6}+\overline{2} \overline{\mathrm{A}} \mathrm{SO}_{3}=\overline{\mathrm{C}} \bar{r}_{2} \mathrm{O}_{4}+\overline{2} \overline{\mathrm{A}} \overline{\mathrm{SO}} \mathrm{O}_{4}
$$

and

$$
\begin{aligned}
\mathrm{Cr}_{2} \mathrm{O}_{5}+{ }_{4} \mathrm{HI}=\mathrm{Cr}_{2} \mathrm{O}_{3}+2 \mathrm{I}_{2}+2 \mathrm{H}_{2} \mathrm{O} ; \text { or, } \\
\\
\qquad \overline{\mathrm{Cr}}_{2} \mathrm{O}_{6}+4 \overline{\mathrm{I}}+4 \stackrel{+}{\mathrm{H}}=\overline{\mathrm{Cr}_{2} \bar{O}_{4}}+2 \mathrm{I}_{2}+2 \mathrm{H}_{2} \mathrm{O}
\end{aligned}
$$

(3) A mixed peroxide of arsenic and chromium of the same degree of oxidation as (I) and (2).

\section{Development of the Theory}

It has been found convenient to introduce the symbol $R p$, for the rate of formation of the hypothetical oxide, according to equations ( $\mathrm{I} 4$ ) or ( $\mathrm{I}_{4} \mathrm{a}$ ), the unit being $0.25 \times 1 \mathrm{IO}^{-5}$ formula-weights if the oxide $\mathrm{As}_{2} \mathrm{O}_{9}$ be assumed, or $0.5 \times 1 \mathrm{IO}^{-5}$ formula-weights if the oxide $\mathrm{Cr}_{2} \mathrm{O}_{5}$ be assumed, and the symbol $R a p$ for the rate of oxidation of arsenious acid by peroxide, according to equations (I 5) and ( $5 a$ ). Rip has already been introduced (page 57): it may now be defined as the rate of oxidation of iodide by peroxide, as in equations (I6) and $(\mathbf{1} 6 a)$.

If the solution contains chromic and arsenious acids only, then for every unit of arsenious acid oxidized in the first stage

1 Although the formula $\mathrm{Cr}_{2} \mathrm{O}_{5}$ contains less oxygen than the formula $\mathrm{CrO}_{3}$ I shall speak of it as a peroxide of chromium, 
(formation of peroxide, equations (I4) and (I $4 a$ )) two more are oxidized in the second stage (reduction of peroxide, equations ( $\mathrm{I}_{5}$ ) and ( $5_{5} a$ )), the final result being the formation of three units of arsenic acid. As, by hypothesis, the second stage takes place instantaneously, the time required for the formation of this amount of arsenic acid is practically that involved in the formation of a unit of peroxide, or,

$$
R p=\frac{I}{3} \cdot R a c
$$

If : however, the solution contains iodide as well as arsenious acid, both will be oxidized on the reduction of the peroxide, the total quantity of iodine and arsenic acid formed in the second stage amounting to two units in all. As before, for one unit of peroxide formed and reduced three units of arsenic acid and iodine taken together are generated, and

$$
R p=\frac{I}{3}(R a+R i p)
$$

Whether iodide be present or not, however, for every unit of peroxide formed une unit of arsenious acid must be oxidized to arsenic acid ${ }^{1}$ any additional arsenic acid formed is due to the oxidation of arsenious acid by the peroxide. Thus,

$$
R a=R p+R a p
$$

It has been pointed out in the paragraph preceding equation (I 8), that two units of iodine and arsenic acid counted together are formed by the reduction of one unit of peroxide; the share of these two units, however, that falls to each, depends upon the relative concentrations of the iodide and arsenious acid. As the concentration of the iodide is increased, the reaction represented by equation (I5) or ( $15 a$ ), falls more and more into the background uritil in the presence of large excess of iodide,

$$
R a p,(K I \text { excess })=0
$$

1 If the peroxide be $\mathrm{Cr}_{2} \mathrm{O}_{5}$, this unit of arsenic acid is formed in the first stage, equation (I $4 a$ ); if $A s_{2} O_{9}$, from the peroxide on reduction, equations (15) and (16). 
whence by equation (19),

$$
R a,(K I \text { excess })=R p
$$

and by equation (I8),

$$
R i p,(K I \text { excess })=2 R p .
$$

Comparison of the Theory with Results of the Experiments

By eliminating the hypothetical $R p$ from equations (17) to (22) the relations deduced from the assumptions of page 77 , may be compared with the experimental results.

The elimination gives:

From equations (I 7 ) and (I8),

$$
R a+R i p=R a c
$$

which is the same as equation (IO); from equations (I7) and (2I),

$$
R a,(\text { KI excess })=\frac{T}{3} \cdot R a c,
$$

which is the same as equation (7); also from equations (2I) and (22),

$$
R i p,(\text { KI excess })=2 R a,(\text { KI excess })=\frac{2}{3} \cdot R a c
$$

which is the same as equation (8).

That is to say, the main quantitative relations between the rates of oxidation of acceptor and inductor are in accord. ance with the postulates of the theory set forth on page 77 . These relations are clearly shown in Fig. 6, which is in obvious agreement with Figs. I to 5, where the curves were plotted from the experiniental data.

\section{Further Development of the Theory}

A graphic representation of the theoretical relations is given in Fig. 6, where the dotted line shows the general form of the Rip curve for increasing concentration of iodide.

Conclusions as to the relative effects of the reagents on the rates, Rap and Rip might be drawn from the form of the Rap 
and Rip curves before they become horizontal; unfortunately, the experimental errors incident to the method of analysis are greatest when the concentration of the iodide is small and this renders any detailed study of the curves useless.

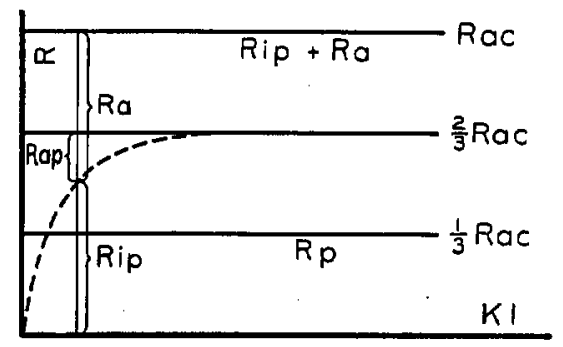

Fig. 6

However, from Tables $\mathrm{V}$ and VI it appears that in solutions containing $A s$ Io, $C r$ 20, $A C$ 242, the rates Rip and $R a p$ are equal when $K I$ is about 25 . If $R i p$ were proportional to the first power of the concentration of the iodide then for $K I=\mathrm{I} 5 \mathrm{O}, \frac{R i p}{R a p}$ would be 6 ; and if to the second power of the iodide, 36 . The first is certainly far too low to be in agreement with the results while the second is not.

Again, comparison of Tables VI and VII shows that when the concentrations of both the arsenic and iodide are doubled the ratio ${ }_{\operatorname{Rap}} i p$ is increased; or in other words the arsenious acid has less effect on the rate $R a p$ than the iodide has on the rate Rip; if Rip be set proportional to the second power of the concentration of the iodide (in accordance with the conclusion reached in last paragraph), then $R a p$ must be set proportional to some lower power of the concentration of the arsenious acid-perhaps the first.

\section{Nature of the Primary 0xide}

As has been shown on page 77 the experimental results of the present paper accord equally well with either of the following assumptions:

(I) A peroxide of arsenic. 
(2) A peroxide of chromium.

(3) A complex peroxide of chromium and arsenic.

If one is to choose between these assumptions, additional evidence is necessary. Such evidence may possibly be obtained in the following ways:

(a) By comparison of the powers of the reagents corresponding to the various hypotheses, with those obtained by experiment-as suggested by Van't Hoff.

Equations (I4) and ( $14 a$ ), corresponding to the assumptions of a peroxide of arsenic and one of chromium respectively both agree with the fact that the rate of oxidation of arsenious acid by chromic acid is proportional to the first power of the concentration of the arsenious acid and to the o.gth power of the concentration of the bichromate (regarding the chromic acid as not being completely dissociated); and equation (I4), together with the view that an ion ${ }^{1}$ of arsenious acid reacts with chromic acid, satisfactorily accounts for the effect of the sulphuric acid concentration, while equation (I $4 a$ ) offers no explanation of the effect of the concentration of the acid. We thus have an argument in favor of assumption (I) or (3), and against assumption (2).

(b) By "systematic variation of the three reagents," as suggested by Luther and Schilow. ${ }^{2}$

Many qualitative experiments were made substituting for chromic acid other oxidizing agents, such as, $\mathrm{H}_{2} \mathrm{O}_{2}, \mathrm{~K}_{2} \mathrm{~S}_{2} \mathrm{O}_{8}$, $\mathrm{KBrO}_{3}, \mathrm{KClO}_{3}, \mathrm{KClO}_{4}$, using starch as indicator for the iodine liberated, and keeping the concentrations of the iodide and sulphuric acid sufficiently large to prevent the arsenious acid from acting on the liberated iodine. In no case was the oxidation of iodide found to be accelerated by the addition of arsenious acid. Thus we have evidence that the primary oxide is a compound of chromium (assumptions (2) and (3)).

Also when certain reducing agents are substituted for acid.

1 See the paper on the rate of oxidation of arsenious acid by chromic 2 Zeit. phys. Chem. 46, 816 (1903). 
arsenious acid the rate of oxidation of iodide by chromic acid is accelerated; this is noticeable in the case of some organic acids as tartaric and oxalic acids ${ }^{1}$ and in the case of ferrous salts $^{2}$ in this last case, however, the acceleration cannot be ascribed to the formation of a peroxide, as Miss Benson has shown. So too, in any given case, to account for the acceleration, different types of intermediate compounds may be assumed in the absence of definite knowledge of the kinetics of the reactions, and it is in this particular that the method suggested by Luther and Schilow fails.

Summing up the evidence brought forward by the application of the above two methods, (a) and (b), we find arguments for and against the assumptions that the primary oxide is one of arsenic or one of chromium, (I) and (2), but none contrary to the view that the primary product is a complex peroxide of arsenic and chromium (3).

\section{Determination of the Degree of Oxidation of a Hypothetical Primary Oxide}

The problem of determining the degree of oxidation of an assumed primary oxide is of general interest, and some methods of doing so will now be given, using the present case in illustration.

(a) From the ratio of the quantities of acceptor and inductor oxidized in any interval (induction factor), when the concentration of the acceptor is so great that this ratio has become constant (as employed by Manchot, Luther and Schilow and others).

Let the composition of the peroxide be represented by the general formula, $A s_{2} \mathrm{O}_{5}+2 r$. Then the reaction between the peroxide and arsenious acid must be written:

$$
A s_{2} O_{5}+{ }_{2} r+r A s_{2} O_{3}=(r+I) A s_{2} O_{5}
$$

and that between the peroxide and the iodide,

$$
\mathrm{As}_{2} \mathrm{O}_{5}-2 r+4 r \mathrm{HI}=2 r \mathrm{I}_{2}+\mathrm{As}_{2} \mathrm{O}_{3}+2 \mathrm{rH}_{2} \mathrm{O} \text {. }
$$

I I have made some measurements on this strbject, and these I hope soon to complete and publish.

"Manchot: loc. cit.; Miss C. C. Benson: loc. cit. 
When the iodide is in excess reaction (23) is negligible, and hence for every equivalent of arsenic acid formed $r$ equivalents of iodine are liberated, or,

$$
\frac{h-i c}{x^{\prime}}(K I \text { excess })=r,
$$

where $h-i c$ and $x^{\prime}$ are the amounts of iodide and of arsenious acid oxidized in the same time (both quatitities being expressed in $c c$ of 0.0 r $N$ solution) see page $6 \mathrm{r}$.

As shown on page $72, r=2$, and hence the peroxide is $\mathrm{As}_{2} \mathrm{O}_{9}$.

If a primary oxide of chromium be assumed the treatment is similar. Regarding $\mathrm{CrO}_{3}$ and $\mathrm{Cr}_{2} \mathrm{O}_{3}$ as the initial and final oxides in the reduction of chromic acid, let the general formula of the peroxide be $\mathrm{Cr}_{2} \mathrm{O}_{3} \div s$. Then for the formation of this oxide we have:

$$
2 \mathrm{CrO}_{3}+\frac{3-s}{2} \mathrm{As}_{2} \mathrm{O}_{3}=\mathrm{Cr}_{2} \mathrm{O}_{3}+\mathrm{s} \div \frac{3-s}{2} \mathrm{As}_{2} \mathrm{O}_{5}
$$

for the oxidation of arsenious acid by peroxide,

$$
\mathrm{Cr}_{2} \mathrm{O}_{3}+s+\frac{s}{2} \mathrm{As}_{2} \mathrm{O}_{3}=\mathrm{Cr}_{2} \mathrm{O}_{3}+\frac{s}{2} \mathrm{As}_{2} \mathrm{O}_{5}
$$

and for the reaction between peroxide and iodide,

$$
\mathrm{Cr}_{2} \mathrm{O}_{3}{ }_{-s}+2 s \mathrm{HI}=\mathrm{Cr}_{2} \mathrm{O}_{3}+s \mathrm{I}_{2} \text {. }
$$

When the iodide is in excess, reaction (27) is negligible, hence,

$$
\frac{h-i c}{x^{\prime}}(K I \text { excess })=\frac{s}{3-s} \text {. }
$$

As shown on page 72 the value of the ratio $(h-i c): x^{\prime}$, when the iodide is in excess, is 2 , hence $s=2$, and the formula of the peroxide is $\mathrm{Cr}_{2} \mathrm{O}_{5}$.

(b) From the ratio of the rates of oxidation of the inductor in the absence of the acceptor to that in the presence of excess of the acceptor.

If the peroxide be one of arsenic, $A s_{2} \mathrm{O}_{5}+2 n$, we have from equations (23) and (24):

$$
\frac{R a c}{R a,(\text { KI excess })}=r+\mathrm{I}, \text { or, } \frac{R a c-R a,(\text { KI excess })}{R a,(K I \text { cxcess })}=r .
$$


Hence from the definitions of $k^{\prime}$ and $k^{\prime}$ given on page 62 .

$$
\frac{k}{k^{\prime}}=r+\mathrm{I}, \text { or, } \frac{k-k^{\prime}}{k^{\prime}}=r
$$

Or if a peroxide of chromium, $\mathrm{Cr}_{2} \mathrm{O}_{3}+s$, be assumed, we have from equations (26) and (27):

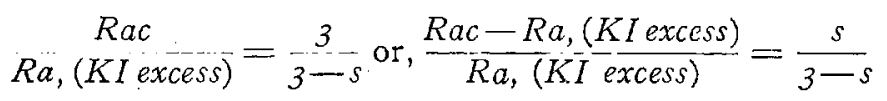

hence also,

$$
\stackrel{k}{k^{\prime}}=\frac{3}{3-s}, \text { or, } \frac{k-k^{\prime}}{k^{\prime}}=\frac{s}{3-s} .
$$

In Tables $\mathrm{V}$-IX it is seen that the value of the ratio $R a c: R a$, (KI excess) is very close to 3 ; hence from equation (30), $r=2$, and from equation (32), s=2. In the summary, Table XXIII: the value of the ratio, $k: k^{\prime}$ is seen to be 3 ; hence again $r$ and $s$ have the value 2 (equations (3I), and (33)). Thus the formula of the peroxide must be written, $\mathrm{As}_{3} \mathrm{O}_{2}$ or $\mathrm{Cr}_{2} \mathrm{O}_{5}$.

(c) From the amounts of the acceptor oxidized in known intervals, assuming that the law of the oxidation of the inductor in the absence of acceptor is known.

It is generally very difficult to determine the amounts of each of two reducing agents in the presence of an oxidizing agent, and quite often impossible to find accurately the induction factor (when the reaction is over); in such cases if it is possible to make near the beginning of the reaction a few measurements of the amount of acceptor oxidized when this reagent is in excess, the degree of oxidation of the primary product may be calculated by the following methods.

The rate of oxidation of arsenious acid by chromic acid is proportional to the first power of the concentration of the arsenious acid; thus using the symbols defined on page 62 , we have,

$$
k=\frac{\mathrm{I}}{t} \cdot \log _{10} \frac{A s}{A s-x}
$$

and when the acceptor is present in excess,

$$
k^{\prime}=\frac{\mathrm{I}}{t} \cdot \log _{10} \frac{A s}{A s-x^{\prime}} .
$$


When the acceptor is in excess, we have, assuming the formula of the peroxide to be $\mathrm{As}_{2} \mathrm{O}_{5}+2 r$

$$
\frac{h-i c}{x^{\prime}}(K I \text { excers })=r
$$

whence, writing $a$ for $h-i c$ to economize space,

$$
x^{\prime}=\frac{a}{r}
$$

and we have,

$$
\frac{T}{t_{1}} \cdot \log _{10} \frac{A s}{A s-\frac{a_{1}}{r}}=\frac{I}{t_{2}} \cdot \log _{10} \frac{A s}{A s-\frac{a_{2}}{r}}=\frac{l}{t_{3}} \cdot \log _{10} \frac{A s}{A s-\frac{a_{3}}{r}}=\ldots
$$

where $t_{1}, a_{1} ; t_{2}, a_{2} ; t_{3}, a_{3}$; etc., are experimentally determined values of $t$ and $a$.

(i) This equation may be solved for $r$, most simply by testing in turn, the numbers, $\mathrm{I}, 2$, etc.

If the rate of oxidation of the inductor had been proportional to any other power of its concentration than the first, an equation somewhat similar to (36) could be written, to be solved for $r$.

(ii) If it be assumed that the value of $k$ in equation (34) be known, $r$ can be found from the following equation (which is obtained from (3I) and (33)):

$$
k^{\prime}=\frac{k}{r+I}=\frac{r}{t} \cdot \log _{10} \frac{A s}{A s-\frac{a}{r}}
$$

by putting it in the form,

$$
\begin{gathered}
\frac{2 \cdot 30 k}{r+\mathrm{I}}=-\frac{I}{t} \cdot \log _{e}\left(I-\frac{a}{r \cdot A s}\right) \\
=\frac{I}{t}\left\{\frac{a}{r \cdot A s}+\frac{I}{2}\left(\frac{a}{r \cdot A s}\right)^{2}+\frac{I}{3}\left(\frac{a}{r \cdot A s}\right)^{3}+\ldots\right\} .
\end{gathered}
$$

If the fraction $\frac{a}{r \cdot A s}$ be very small we have as a first approximation,

$$
\frac{2.30 k}{r+r}=\frac{r}{t} \cdot \frac{a}{r \cdot A s}
$$


and since $a=h-i c$ we have,

$$
r=\frac{0.4343(h-i c)}{k . t . A s-0.4343(h-i c)}=r^{\prime} \text { of Table XXII. }
$$

Using this approximation the values of $r^{\prime}$ given in Table XXII were calculated. It will be noticed that the value of $r^{\prime}$ diminishes as $a(i . e ., h-i c)$. increases, because the neglected terms of equation ( 38$)$ become larger and larger. Hence a closer approximation is to be desired:

$$
\frac{2.30 k}{r+I}=\frac{I}{t}\left(\frac{a}{r \cdot A s}+\frac{I}{t}\left(\frac{a}{r \cdot A s}\right)^{2}\right)
$$

and hence,

$$
r=\frac{a}{4 \cdot A s} \cdot \frac{\sqrt{(a+2 . A s)^{2}+8 \cdot A s(2 \cdot 30 k \cdot t \cdot A s-a)}+(a+2 . A s)}{2 \cdot 30 k \cdot t \cdot A s-a}=r^{\prime \prime}
$$

of Table XXII.

The values of $r^{\prime \prime}$ in Table XXII were calculated by means of formula (42).

These values do not fall off so noticeably on increasing the value of $a(i . e ., h-i c)$ as do the values of $r^{\prime}$ in the same table. Both $r^{\prime}$ and $r^{\prime \prime}$ point to the number 2 as the value of $r$. A slight error in $k$ or $a$ will cause a considerable error in the value of $r^{\prime \prime}$; this is noticeable in initial values of some of the tables.

(iii) The value of $r$ may be found also in the following manner :

We have

$$
\frac{d x^{\prime}}{d t}=2 \cdot 30 k^{\prime}\left(A s--x^{\prime}\right),
$$

and since $a=r \cdot x^{\prime}$, it follows that

$$
\frac{d a}{d t}=2.30 k^{\prime}(r \cdot A s-a)
$$

whence,

and therefure,

$$
a=2.30 k^{\prime} \int_{0}^{t}(r . A s-a) \cdot d t=2.30 k^{\prime}\left(r . A s . t-\int_{0}^{t} a . d t\right),
$$

$$
\frac{a}{t}=2.30 k^{\prime}\left(r . A s-\frac{T}{t} \int_{0}^{t} a \cdot d t\right)
$$


Now the values of $\int_{0}^{t} a \cdot d t$ can be found easily and accurately from the plotted curve, $a, t$, and hence the values of $k^{\prime}$ and of r.As can be found by means of equation (44) for any two pairs of values of $a$ and $t$.

Thus, we have

$$
\frac{r \cdot A s}{A s}=\frac{h-i c}{x^{\prime}}(\text { for } t=-\infty)=r^{1} .
$$

If in addition the value of $k$ of equation (34) be known, the value of $r$ can be determined from the above calculated value of $k^{\prime}$ as in equation (3I).

\section{SUMIMARY}

A method of analysis, which may be of use in the study of other complicated cases of induced oxidation, is described.

Addition of potassium iodide lessens the rate of oxidation of arsenious acid by chromic acid, the retardation increasing with the concentration of the iodide up to a certain point from which on the rate of oxidation of arsenious acid is equal to one-third the rate when no iodide is present; or symbolically,

$$
R a(\text { KI excess })=\frac{I}{3} \cdot \text { Rac. }
$$

The rate at which iodine is liberated it solutions containing arsenious acid, iodide and chromic acid, after correcting for the direct oxidation of iodide by chromic acid, in-

1 As an example of this method the following table calculated from the lata of 'Table XI, by methods described in Jour. Phys. Chem., ro, 423 (I906) is given.

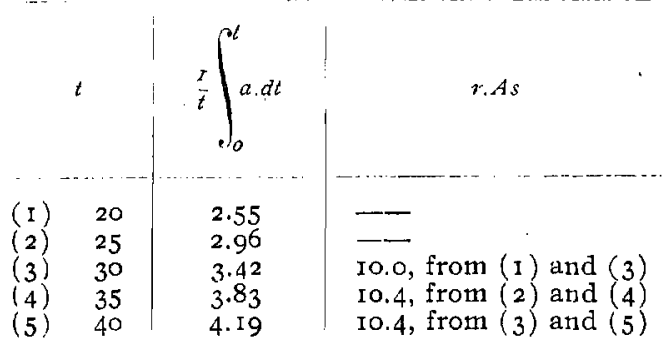

In this table $a=h-i c$ (calc.) of Table XI. The value of $A s$ is 5.00 and hence $r=2$ very closely. 
creases with the concentration of the iodide up to a certain point from which on the rate of liberation of iodine is twice the rate of oxidation of arsenious acid (expressing both iodide and arsenious acid in equivalents), or,

$$
R i p=2 R a,(K I \text { excess). }
$$

The rate of reduction of chromic acid (after. correcting for the direct action of the iodide) is the same for all concentrations of iodide from zero up, or,

$$
R a+R i-R i c=R a+R i p=R a c .
$$

Expressing each of the three rates, Rac (rate of oxidation of arsenious acid by chromic acid in the absence of iodide), $R a$ (rate of oxidation of arsenious acid in the presence of iodide) and Rip (rate of liberation of iodine after correcting for the direct action of the chromic acid on iodide), in terms of the concentrations of the reagents, we have

$$
\begin{aligned}
& R a c=k \cdot A s \cdot(C r)^{\circ} 9 \cdot(A c)^{\mathrm{r} \cdot 4} \\
& R a=k^{\prime} \cdot A s \cdot(C r)^{\circ 9} \cdot(A c)^{\mathrm{r} .4} \\
& R i p=k^{\prime \prime} \cdot A s \cdot(C r)^{\circ .9} \cdot(A c)^{\mathrm{r} .4}
\end{aligned}
$$

where $k^{\prime}$ and $k^{\prime \prime}$ depend on the concentration of the iodide. When the iodide is in excess,

$$
k=3 k^{\prime}=\frac{3}{2} \cdot k^{\prime \prime} .
$$

The effect of the temperature on the rates $R a c, R a$ ( $K I$ excess) and Rip, ( $K I$ excess) is the same, the coefficient being unusually small (somewhat less than I.3).

The results may be accounted for by assuming:

(a) That arsenious acid acting on chromic acid forms slowly a primary oxide.

(b) That this oxide is completely and instantaneously reduced by either arsenious acid or iodide.

(c) That the fractions of the primary oxide reduced by arsenious acid and iodide respectively, in solutions containing both, depend on their relative concentrations: when the iodide is in large excess the amount of the primary oxide reduced by the arsenious acid is negligible. 
The primary oxide may be one of the following:

(I) A peroxide of arsenic represented by the formula, $A s_{2} O_{8}$, or one of its hydrates, e. g., $H_{3} A s O_{6}$.

(2) An intermediate oxide of chromium of the formula, $\mathrm{Cr}_{2} \mathrm{O}_{5}$, or one of its hydrates, e. g., $\mathrm{H}_{2} \mathrm{Cr}_{2} \mathrm{O}_{6}$.

(3) A complex peroxide of arsenic and chromium of the same degree of oxidation as (I) and (2).

Other evidence is in support of the view that the peroxide is one of arsenic; and still other evidence favors the assumption that the primary oxide is a compound of chromium; the view that the primary oxide is a complex peroxide of arsenic and chromium is, however, in best accord with all the facts.

Methods are described by which the degree of oxidation of a hypothetical primary oxide may be determined.

The above experimental work was completed in the Chemical Laboratory of the University of Toronto in May, I 905 .

I wish here to express my thanks to Professor W. Lash Miller at whose suggestion this research was undertaken, for his kind advice and assistance throughout.

CONTENTS

IN CRODUCTION. PLAN OF THE INVESTIGATION

EXPERIMENTAL PART. METHOD, SOLUTIONS, EXPLANATION OF THE

TABI.ES, TABLES

DISCUSSION OF THE EXPERIMENTAL DATA. EFFECT OF THE CONCENI'RATION OF IODIDE, ARSENIOUS ACID, BICHROMATE AND ACID.

EFFECT OF TEMTERATURE.

EORETICAL PART. PEROXIDE THEORY, ASSUMPTIONS, COMPARISON WITH EXPERIMENTAI, RESUI,TS. NATURE OF THE PRIMARY OXIDE... 76 DETERMINATION OF THE DEGREES OF OXIDATION OF A HYPOTHETICAL

PRIMARY OXIDE. 